\title{
Fair Endowment Calculation for Nursing Homes Using Actuarial Techniques: A Turkey Experience
}

\author{
Aktüeryal Teknikleri Kullanılarak Huzurevleri İçin Adil Bağış Hesaplaması: \\ Türkiye Örneği
}

\begin{abstract}
Background: Nursing homes continue to play an important role in elderly services, including offering physical, psychological, and social support. For this reason, it is crucial to ensure their sustainable financial management. Aims: This study aims to build a model that incorporates factors such as age, gender, and real rate of return, all of which affect the minimum amount of the one-time endowment. Methods: In this study, the minimum endowment is calculated using actuarial techniques by considering the age and gender of the nursing home resident along with the real rate of return for endowments. Our model incorporates a probability of spending calculated using mortality rates from Turkey Life Tables (TRH2010) and a 2\% real rate of return. Results: The expected value of $¥ 1$ spent each year as long as the individual lives varies with age and gender. For a 60-year-old female, this expected value is over 0.99 (i.e., 99\% probability of spending $\equiv 1$ during the year), whereas it falls below 0.50 for an 81-year-old, and 0.10 for a 90-year-old. For a 60-year-old male, the expected value is about 0.99, which falls below 0.50 for a 78-year-old, and 0.10 for an 89-year-old. Thus, the customary endowment is insufficient for female elderly persons below the age of 71 and male elderly persons below the age of 68. Conclusion: Many factors can affect the fair amount of a one-time endowment. Failure to take these factors into account may seriously jeopardize the fairness and sustainability of elderly services.
\end{abstract}

Öz

Arka plan: Huzurevleri, fiziksel, psikolojik ve sosyal destek de dâhil olmak üzere yaşl hizmetlerinde önemli bir rol oynar. Bu nedenle, huzurevlerinin sürdürülebilir finansal yönetimlerini sağlamak çok önemlidir. Amaç: Bu çalışma, huzurevlerinde tek seferlik bă̆ış miktarının adil ve doğru belirlenmesinde yaş, cinsiyet ve gerçek getiri oranı gibi faktörleri içeren bir model oluşturmayı amaçlamaktadır. Yöntem: Bu çalışmada asgari bağış, huzurevinde ikamet eden kişinin yaşı ve cinsiyeti ile bağışların reel getiri oranı dikkate alınarak aktüeryal teknikler kullanılarak hesaplanmıştır. Modelimiz, Türkiye Yaşam Tablolarından (TRH-2010) alınan ölüm oranları kullanılarak hesaplanan bir harcama olasılığını ve\% 2'lik bir reel getiri oranını içermektedir. Bulgular: Bireysel yaşamlar yaşa ve cinsiyete göre değiştiği sürece her yıl harcanan 1 TL'nin beklenen değgeri. 60 yaşında bir kadın için bu beklenen değer 0,99'un üzerindedir (yani yil içinde 1 TL harcama olasilığı\% 99), 81 yaşında ise 0,50' nin ve 90 yaşında 0,10'un altına düşmektedir. 60 yaşında bir erkek için beklenen değer yaklaşık 0,99'dur ve 78 yaşında birisi için 0,50' nin ve 89 yaşında birisi için 0,10'un altına düşmektedir. Bu nedenle, 71 yaşın altındaki kadın yaşlılar ve 68 yaşın altındaki erkek yaşlılar için geleneksel bağış yetersizdir. Sonuç: Bir kerelik bağışın adil miktarın birçok faktör etkileyebilir. Bu faktörlerin hesaba katılmaması, yaşlı hizmetlerinin adaletini ve sürdürülebilirliğini tehlikeye atabilir.

\author{
İlhan Kerem Şenel \\ Prof. Dr, Istanbul University- \\ Cerrahpasa, Faculty of Health Sciences, \\ Health Management, \\ keremsenel@gmail.com, \\ Orcid: 0000-0003-4496-5149
}

\section{Canser Boz}

Dr. Arş. Gör., Istanbul University-

Cerrahpasa, Faculty of Health Sciences,

Health Economics,

canser.boz@istanbul.edu.tr,

Orcid: 0000-0002-6136-4479

\author{
Ahmet Akgül \\ Prof. Dr, Istanbul University- \\ Cerrahpasa, Faculty of Health Sciences, \\ Health Management, \\ aakgul@hotmail.com, \\ Orcid: 0000-0002-8399-7090
}

\section{Musa Çırak}

Doç. Dr., Istanbul Bakıköy Dr. Sadi

Konuk Ĕ̆itim ve Araştırma Hastanesi, musacirak@hotmail.com,

Orcid: 0000-0002-0175-9655

Article Type / Makale Türü

Research Article / Araştırma Makalesi

Anahtar Kelimeler

Huzurevleri, Aktüeryal Teknikler, Bă̆ıs,

Ölüm, Yaşl

\section{Keywords}

Nursing Homes, Actuarial Techniques, Endowment, Mortality, Elderly

JEL Codes: I1,C6, G0

Submitted: $\quad 15$ / 02 / 2021

Accepted: $\quad 07 / 04 / 2021$ 


\section{Introduction}

Old age is not a new phenomenon, but many societies are witnessing a steadily rising average age, leading to issues regarding sustainability of the government pension system as well as staggering healthcare costs. Spending programs for the elderly are at the forefront of the problems faced by both developed and developing countries. For this reason, countries are developing new policy proposals to reduce the cost of aging, improve the elderly's quality of health, and avoid social security vulnerabilities (Gokbunar et all, 2016)

In recent years, despite the proliferation of home care services and living spaces, private alternative policies and practices are emerging. Nursing homes, the first line of elderly services in Turkey, date back to Seljuk origin (circa 100 A.D.). They still play an important role within the framework of social security and social services programs for the elderly (Ardahan, 2010).

Old age leads to many problems such as chronic diseases, disabilities, and dependence on other people. Elderly people can suffer from the loss of physical and mental capacity, become dependent on others, and face increasing psychological problems. They might fail to take their prescribed medicine regularly and might be unable to perform adequate personal care due to chronic diseases and disabilities (Lau et all, 2005). This may cause the elderly to be dependent on others for personal and medical care, to the point of possibly requiring institutions or individuals to care for them. Nursing home care involves ensuring the continuity of the physical, mental, and social capacity of elderly residents for as long as possible (Wagner et all, 2001). In addition, nursing homes should not only provide clinical care but also meet the social and psychological needs of their residents (Glass, 1991). For this reason, it is crucial to ensure sustainable financial management for these nursing homes.

Nursing homes have been affected by neoliberal economic movements that emerged globally at the beginning of 1980s, and they have been increasingly financed by the private sector rather than the public sector. This is a natural consequence of the demand for higher quality services. Nursing home services that have primarily been funded by the public sector have progressed toward a structure in which costs are increasingly covered by individuals. As a result, individuals have started to purchase insurance to pay for these expenses (Çadır, 2017).

Elderly people accepted to nursing homes have two payment options (Turkey Official Newspaper, 2001). The first option is monthly payments, the amount of which is determined by the Ministry of Family and Social Policies. The monthly fee received from an elderly person residing in a single room in a nursing home operated by Kizilay (Turkish Red Crescent) is £1,750 for 2017 excluding VAT. This fee was used as the monthly fee in our study. The second option is a one-time endowment that is expected to compensate for the care costs of the elderly person for the rest of her/his life. In 2017, a nursing home operated by Kizilay required a one-time endowment equivalent to an upfront payment for 10 years.

This study aims to build a model that incorporates factors such as age, gender, and real rate of return, all of which affect the minimum amount of the one-time endowment.

\section{Materials and Method}

\subsection{Method of Study}

The minimum amount of endowment needed is calculated using actuarial techniques and considering the age and gender of the elderly person as well as the real rate of return for endowments. Our model incorporates the probability of spending calculated using mortality rates from Turkey Life Tables (TRH-2010) and a 2\% real rate of return. We utilized R 3.4.2 and R Studio Desktop 1.1.442 software packages for our computations. The R script is included in Appendix A.

\subsection{Limitations}

Apart from these three factors, estimates of an individual's current and future health status can also affect the costs and, therefore, the minimum amount of endowment. For example, the cost of a bedridden patient and cost of an elderly person able to self-care will be very different. As another example, the life expectancy of a patient who has had a heart attack will naturally be shorter than that of a healthy individual. In addition, extensive data are needed to study the impact of these 
factors. We plan to incorporate some of these factors into our model in our future studies due to and the availability of appropriate data and the scope of this study.

\subsection{Factors Affecting Minimum Endowment Amount}

Age: This factor indicates the age at which the elderly person is admitted to the nursing home, which is the most important factor affecting the minimum endowment amount. The life expectancy of a person aged 60 and another person aged 90 are very different. According to Turkey Life Tables (TRH-2010), the life expectancy of a 60-year-old female is 20.79 years whereas the life expectancy of a 90-year-old is only 3.29 years (Hacettepe University, 2010).

Gender: Women live longer than men on average. According to WHO data, in 2015, the life expectancy at birth is 73.7 years for women and 69.1 years for men. In Europe, which has the longest life expectancy in the world, the difference is even higher. Life expectancy at birth in Europe is 80.2 years for women but merely 73.2 years for men (WHO,2016).

According to Turkey Life Tables (TRH-2010), the life expectancy of a 60-year-old woman is 20.79 years, whereas the life expectancy of a male of the same age is 17.62 years. This difference is more important for individuals who are admitted to nursing homes at a relatively young age, albeit with a declining difference in life expectancies at higher ages.

Real Rate of Return: As the real rate of return increases, the initial endowment will be invested at a higher rate, and the minimum amount expected to compensate for the care costs of the elderly person for the rest of her/his life turn out to be lower. The real rate of return is assumed to be $2 \%$. For future studies, we plan to conduct a sensitivity analysis to determine the impact of the real rate of return on the minimum amount of initial endowment. For instance, historical data covering the last 10 years may be utilized to get a more realistic estimate of the real rate of return. Hence, despite having the real rate of return as a variable in our model, our current study focuses on the impact of age and gender.

\section{Results}

\subsection{Life Expectancy Calculations}

Turkey Female and Males Life Tables (TRH-2010) are given in Table 1. Turkey Female Life (TRH-2010) and Table 2. Turkey Male Life (TRH-2010), respectively. The explanations of the symbols in the table are as follows (Strauss and Shavelle, 2010).

Table 1. Turkey Female Life (TRH-2010)

\begin{tabular}{|c|c|c|c|c|c|c|c|c|c|c|c|c|c|}
\hline Age $x$ & $q_{x}$ & $p_{x}$ & $1_{x}$ & $d_{x}$ & $\mathbf{e}_{\mathrm{x}}$ & $\overline{e_{x} \text { at } x}$ & Age $x$ & $q_{x}$ & $p_{x}$ & $1_{x}$ & $d_{x}$ & $e_{x}$ & $\overline{e_{x} \text { at } x}$ \\
\hline 0 & 0.008161 & 0.991839 & $100,000.00$ & 816.11 & 78.02 & 78.02 & 50 & 0.002650 & 0.997350 & $96,638.68$ & 256.06 & 29.74 & 79.74 \\
\hline 1 & 0.000278 & 0.999722 & $99,183.89$ & 27.57 & 77.66 & 78.66 & 51 & 0.002840 & 0.997160 & $96,382.62$ & 273.75 & 28.82 & 79.82 \\
\hline 2 & 0.000235 & 0.999765 & $99,156.31$ & 23.32 & 76.68 & 78.68 & 52 & 0.003129 & 0.996871 & $96,108.87$ & 300.72 & 27.90 & 79.90 \\
\hline 3 & 0.000202 & 0.999798 & $99,132.99$ & 20.07 & 75.70 & 78.70 & 53 & 0.003517 & 0.996483 & $95,808.16$ & 336.94 & 26.98 & 79.98 \\
\hline 4 & 0.000187 & 0.999813 & $99,112.92$ & 18.50 & 74.72 & 78.72 & 54 & 0.004006 & 0.995994 & $95,471.21$ & 382.44 & 26.08 & 80.08 \\
\hline 5 & 0.000143 & 0.999857 & $99,094.41$ & 14.17 & 73.73 & 78.73 & 55 & 0.004401 & 0.995599 & $95,088.77$ & 418.45 & 25.18 & 80.18 \\
\hline 6 & 0.000116 & 0.999884 & $99,080.25$ & 11.53 & 72.74 & 78.74 & 56 & 0.004735 & 0.995265 & $94,670.32$ & 448.28 & 24.29 & 80.29 \\
\hline 7 & 0.000100 & 0.999900 & $99,068.72$ & 9.89 & 71.75 & 78.75 & 57 & 0.005225 & 0.994775 & $94,222.04$ & 492.34 & 23.40 & 80.40 \\
\hline 8 & 0.000093 & 0.999907 & $99,058.82$ & 9.26 & 70.76 & 78.76 & 58 & 0.005875 & 0.994125 & $93,729.70$ & 550.63 & 22.52 & 80.52 \\
\hline 9 & 0.000097 & 0.999903 & $99,049.57$ & 9.62 & 69.76 & 78.76 & 59 & 0.006688 & 0.993312 & $93,179.07$ & 623.14 & 21.65 & 80.65 \\
\hline 10 & 0.000094 & 0.999906 & $99,039.95$ & 9.31 & 68.77 & 78.77 & 60 & 0.007251 & 0.992749 & $92,555.94$ & 671.16 & 20.79 & 80.79 \\
\hline 11 & 0.000084 & 0.999916 & $99,030.64$ & 8.34 & 67.78 & 78.78 & 61 & 0.007724 & 0.992276 & $91,884.78$ & 709.70 & 19.94 & 80.94 \\
\hline 12 & 0.000084 & 0.999916 & $99,022.30$ & 8.37 & 66.78 & 78.78 & 62 & 0.008609 & 0.991391 & $91,175.08$ & 784.97 & 19.09 & 81.09 \\
\hline 13 & 0.000095 & 0.999905 & $99,013.94$ & 9.39 & 65.79 & 78.79 & 63 & 0.009923 & 0.990077 & $90,390.11$ & 896.96 & 18.26 & 81.26 \\
\hline 14 & 0.000115 & 0.999885 & $99,004.55$ & 11.41 & 64.79 & 78.79 & 64 & 0.011685 & 0.988315 & $89,493.14$ & $1,045.69$ & 17.43 & 81.43 \\
\hline 15 & 0.000135 & 0.999865 & $98,993.13$ & 13.37 & 63.80 & 78.80 & 65 & 0.013220 & 0.986780 & $88,447.45$ & $1,169.28$ & 16.63 & 81.63 \\
\hline 16 & 0.000148 & 0.999852 & $98,979.76$ & 14.66 & 62.81 & 78.81 & 66 & 0.014533 & 0.985467 & $87,278.18$ & $1,268.37$ & 15.85 & 81.85 \\
\hline 17 & 0.000162 & 0.999838 & $98,965.11$ & 16.05 & 61.82 & 78.82 & 67 & 0.016337 & 0.983663 & $86,009.80$ & $1,405.18$ & 15.08 & 82.08 \\
\hline 18 & 0.000177 & 0.999823 & $98,949.06$ & 17.54 & 60.83 & 78.83 & 68 & 0.018672 & 0.981328 & $84,604.62$ & $1,579.70$ & 14.32 & 82.32 \\
\hline 19 & 0.000193 & 0.999807 & $98,931.51$ & 19.14 & 59.84 & 78.84 & 69 & 0.021583 & 0.978417 & $83,024.92$ & $1,791.93$ & 13.58 & 82.58 \\
\hline 20 & 0.000210 & 0.999790 & $98,912.37$ & 20.76 & 58.85 & 78.85 & 70 & 0.024463 & 0.975537 & $81,232.99$ & $1,987.24$ & 12.87 & 82.87 \\
\hline 21 & 0.000226 & 0.999774 & $98,891.62$ & 22.31 & 57.86 & 78.86 & 71 & 0.027224 & 0.972776 & $79,245.75$ & $2,157.41$ & 12.18 & 83.18 \\
\hline 22 & 0.000241 & 0.999759 & $98,869.30$ & 23.83 & 56.88 & 78.88 & 72 & 0.030523 & 0.969477 & $77,088.34$ & $2,352.96$ & 11.51 & 83.51 \\
\hline 23 & 0.000256 & 0.999744 & $98,845.48$ & 25.30 & 55.89 & 78.89 & 73 & 0.034440 & 0.965560 & $74,735.38$ & $2,573.88$ & 10.85 & 83.85 \\
\hline 24 & 0.000271 & 0.999729 & $98,820.18$ & 26.73 & 54.90 & 78.90 & 74 & 0.039082 & 0.960918 & $72,161.50$ & $2,820.20$ & 10.22 & 84.22 \\
\hline 25 & 0.000282 & 0.999718 & $98,793.45$ & 27.90 & 53.92 & 78.92 & 75 & 0.044930 & 0.955070 & $69,341.30$ & $3,115.50$ & 9.62 & 84.62 \\
\hline 26 & 0.000295 & 0.999705 & $98,765.55$ & 29.09 & 52.93 & 78.93 & 76 & 0.051247 & 0.948753 & $66,225.81$ & $3,393.88$ & 9.05 & 85.05 \\
\hline
\end{tabular}




\begin{tabular}{|c|c|c|c|c|c|c|c|c|c|c|c|c|c|}
\hline 27 & 0.000311 & 0.999689 & $98,736.46$ & 30.68 & 51.95 & 78.95 & 77 & 0.057276 & 0.942724 & $62,831.93$ & $3,598.77$ & 8.51 & 85.51 \\
\hline 28 & 0.000331 & 0.999669 & $98,705.77$ & 32.69 & 50.97 & 78.97 & 78 & 0.062975 & 0.937025 & $59,233.16$ & $3,730.18$ & 8.00 & 86.00 \\
\hline 29 & 0.000356 & 0.999644 & $98,673.09$ & 35.09 & 49.98 & 78.98 & 79 & 0.068250 & 0.931750 & $55,502.98$ & $3,788.10$ & 7.50 & 86.50 \\
\hline 30 & 0.000372 & 0.999628 & $98,638.00$ & 36.67 & 49.00 & 79.00 & 80 & 0.075940 & 0.924060 & $51,714.88$ & $3,927.21$ & 7.01 & 87.01 \\
\hline 31 & 0.000385 & 0.999615 & $98,601.33$ & 37.98 & 48.02 & 79.02 & 81 & 0.086117 & 0.913883 & $47,787.67$ & $4,115.31$ & 6.55 & 87.55 \\
\hline 32 & 0.000411 & 0.999589 & $98,563.35$ & 40.53 & 47.04 & 79.04 & 82 & 0.095750 & 0.904250 & $43,672.36$ & $4,181.63$ & 6.12 & 88.12 \\
\hline 33 & 0.000450 & 0.999550 & $98,522.82$ & 44.32 & 46.06 & 79.06 & 83 & 0.104485 & 0.895515 & $39,490.73$ & $4,126.17$ & 5.71 & 88.71 \\
\hline 34 & 0.000501 & 0.999499 & $98,478.50$ & 49.35 & 45.08 & 79.08 & 84 & 0.111664 & 0.888336 & $35,364.56$ & $3,948.94$ & 5.32 & 89.32 \\
\hline 35 & 0.000536 & 0.999464 & $98,429.15$ & 52.71 & 44.10 & 79.10 & 85 & 0.122720 & 0.877280 & $31,415.62$ & $3,855.31$ & 4.93 & 89.93 \\
\hline 36 & 0.000562 & 0.999438 & $98,376.44$ & 55.25 & 43.12 & 79.12 & 86 & 0.139435 & 0.860565 & $27,560.31$ & $3,842.87$ & 4.54 & 90.54 \\
\hline 37 & 0.000613 & 0.999387 & $98,321.19$ & 60.29 & 42.15 & 79.15 & 87 & 0.156215 & 0.843785 & $23,717.43$ & $3,705.02$ & 4.20 & 91.20 \\
\hline 38 & 0.000690 & 0.999310 & $98,260.91$ & 67.83 & 41.17 & 79.17 & 88 & 0.171981 & 0.828019 & $20,012.42$ & $3,441.75$ & 3.88 & 91.88 \\
\hline 39 & 0.000793 & 0.999207 & $98,193.08$ & 77.88 & 40.20 & 79.20 & 89 & 0.184245 & 0.815755 & $16,570.67$ & $3,053.07$ & 3.59 & 92.59 \\
\hline 40 & 0.000860 & 0.999140 & $98,115.19$ & 84.41 & 39.23 & 79.23 & 90 & 0.196392 & 0.803608 & $13,517.60$ & $2,654.75$ & 3.29 & 93.29 \\
\hline 41 & 0.000911 & 0.999089 & $98,030.78$ & 89.27 & 38.26 & 79.26 & 91 & 0.215417 & 0.784583 & $10,862.85$ & $2,340.04$ & 2.97 & 93.97 \\
\hline 42 & 0.001015 & 0.998985 & $97,941.51$ & 99.41 & 37.30 & 79.30 & 92 & 0.239333 & 0.760667 & $8,522.81$ & $2,039.79$ & 2.64 & 94.64 \\
\hline 43 & 0.001174 & 0.998826 & $97,842.10$ & 114.83 & 36.34 & 79.34 & 93 & 0.270550 & 0.729450 & $6,483.02$ & $1,753.98$ & 2.32 & 95.32 \\
\hline 44 & 0.001387 & 0.998613 & $97,727.27$ & 135.54 & 35.38 & 79.38 & 94 & 0.313514 & 0.686486 & $4,729.04$ & $1,482.62$ & 1.99 & 95.99 \\
\hline 45 & 0.001574 & 0.998426 & $97,591.73$ & 153.57 & 34.43 & 79.43 & 95 & 0.370141 & 0.629859 & $3,246.42$ & $1,201.63$ & 1.67 & 96.67 \\
\hline 46 & 0.001725 & 0.998275 & $97,438.16$ & 168.07 & 33.48 & 79.48 & 96 & 0.445527 & 0.554473 & $2,044.79$ & 911.01 & 1.36 & 97.36 \\
\hline 47 & 0.001918 & 0.998082 & $97,270.09$ & 186.60 & 32.54 & 79.54 & 97 & 0.559928 & 0.440072 & $1,133.78$ & 634.83 & 1.05 & 98.05 \\
\hline 48 & 0.002154 & 0.997846 & $97,083.49$ & 209.13 & 31.60 & 79.60 & 98 & 0.747798 & 0.252202 & 498.95 & 373.11 & 0.75 & 98.75 \\
\hline 49 & 0.002433 & 0.997567 & $96,874.36$ & 235.68 & 30.67 & 79.67 & 99 & 1.000000 & 0.000000 & 125.84 & 125.84 & 0.50 & 99.50 \\
\hline
\end{tabular}

Table 2. Turkey Male Life (TRH-2010)

\begin{tabular}{|c|c|c|c|c|c|c|c|c|c|c|c|c|c|}
\hline \multirow[b]{2}{*}{ Age $x$} & \multirow[b]{2}{*}{$q_{x}$} & \multirow[b]{2}{*}{$p_{x}$} & \multirow[b]{2}{*}{$l_{x}$} & \multirow[b]{2}{*}{$d_{x}$} & \multirow[b]{2}{*}{$e_{x}$} & $e_{x}$ at $x$ & \multirow{2}{*}{- Age } & \multirow[b]{2}{*}{$q_{x}$} & \multirow[b]{2}{*}{$p_{x}$} & \multirow[b]{2}{*}{$\mathbf{l}_{\mathrm{x}}$} & \multirow[b]{2}{*}{$d_{x}$} & \multirow[b]{2}{*}{$\mathbf{e}_{x}$} & \multirow{2}{*}{$\begin{array}{c}e_{x} \text { at } \\
x \\
\end{array}$} \\
\hline & & & & & & & & & & & & & \\
\hline 0 & 0.019533 & 0.980467 & $100,000.00$ & $1,953.31$ & 71.93 & 71.93 & 50 & 0.005159 & 0.994841 & $92,248.01$ & 475.94 & 25.79 & 75.79 \\
\hline 1 & 0.000888 & 0.999112 & $98,046.69$ & 87.08 & 72.35 & 73.35 & 51 & 0.005558 & 0.994442 & $91,772.07$ & 510.08 & 24.93 & 75.93 \\
\hline 2 & 0.000776 & 0.999224 & $97,959.61$ & 76.03 & 71.42 & 73.42 & 52 & 0.006163 & 0.993837 & $91,261.98$ & 562.44 & 24.06 & 76.06 \\
\hline 3 & 0.000686 & 0.999314 & $97,883.58$ & 67.17 & 70.47 & 73.47 & 53 & 0.006979 & 0.993021 & $90,699.55$ & 633.00 & 23.21 & 76.21 \\
\hline 4 & 0.000642 & 0.999358 & $97,816.41$ & 62.84 & 69.52 & 73.52 & 54 & 0.008014 & 0.991986 & $90,066.55$ & 721.77 & 22.37 & 76.37 \\
\hline 5 & 0.000552 & 0.999448 & $97,753.58$ & 54.00 & 68.57 & 73.57 & 55 & 0.008968 & 0.991032 & $89,344.78$ & 801.27 & 21.54 & 76.54 \\
\hline 6 & 0.000430 & 0.999570 & $97,699.57$ & 42.00 & 67.60 & 73.60 & 56 & 0.009810 & 0.990190 & $88,543.51$ & 868.63 & 20.74 & 76.74 \\
\hline 7 & 0.000355 & 0.999645 & $97,657.57$ & 34.64 & 66.63 & 73.63 & 57 & 0.010834 & 0.989166 & $87,674.87$ & 949.90 & 19.94 & 76.94 \\
\hline 8 & 0.000327 & 0.999673 & $97,622.92$ & 31.92 & 65.66 & 73.66 & 58 & 0.012050 & 0.987950 & $86,724.97$ & $1,045.07$ & 19.15 & 77.15 \\
\hline 9 & 0.000347 & 0.999653 & $97,591.00$ & 33.84 & 64.68 & 73.68 & 59 & 0.013470 & 0.986530 & $85,679.90$ & $1,154.15$ & 18.38 & 77.38 \\
\hline 10 & 0.000335 & 0.999665 & $97,557.16$ & 32.66 & 63.70 & 73.70 & 60 & 0.014781 & 0.985219 & $84,525.75$ & $1,249.42$ & 17.62 & 77.62 \\
\hline 11 & 0.000291 & 0.999709 & $97,524.50$ & 28.40 & 62.72 & 73.72 & 61 & 0.016036 & 0.983964 & $83,276.33$ & $1,335.41$ & 16.88 & 77.88 \\
\hline 12 & 0.000295 & 0.999705 & $97,496.10$ & 28.77 & 61.74 & 73.74 & 62 & 0.017600 & 0.982400 & $81,940.92$ & $1,442.13$ & 16.14 & 78.14 \\
\hline 13 & 0.000347 & 0.999653 & $97,467.33$ & 33.78 & 60.76 & 73.76 & 63 & 0.019498 & 0.980502 & $80,498.79$ & $1,569.56$ & 15.42 & 78.42 \\
\hline 14 & 0.000446 & 0.999554 & $97,433.56$ & 43.43 & 59.78 & 73.78 & 64 & 0.021763 & 0.978237 & $78,929.24$ & $1,717.70$ & 14.72 & 78.72 \\
\hline 15 & 0.000551 & 0.999449 & $97,390.13$ & 53.71 & 58.80 & 73.80 & 65 & 0.024068 & 0.975932 & $77,211.54$ & $1,858.30$ & 14.04 & 79.04 \\
\hline 16 & 0.000626 & 0.999374 & $97,336.42$ & 60.90 & 57.84 & 73.84 & 66 & 0.026344 & 0.973656 & $75,353.23$ & $1,985.10$ & 13.37 & 79.37 \\
\hline 17 & 0.000690 & 0.999310 & $97,275.52$ & 67.14 & 56.87 & 73.87 & 67 & 0.028939 & 0.971061 & $73,368.13$ & $2,123.20$ & 12.72 & 79.72 \\
\hline 18 & 0.000745 & 0.999255 & $97,208.38$ & 72.42 & 55.91 & 73.91 & 68 & 0.031899 & 0.968101 & $71,244.93$ & $2,272.62$ & 12.08 & 80.08 \\
\hline 19 & 0.000790 & 0.999210 & $97,135.96$ & 76.76 & 54.95 & 73.95 & 69 & 0.035280 & 0.964720 & $68,972.31$ & $2,433.34$ & 11.47 & 80.47 \\
\hline 20 & 0.000858 & 0.999142 & $97,059.20$ & 83.24 & 53.99 & 73.99 & 70 & 0.039232 & 0.960768 & $66,538.97$ & $2,610.44$ & 10.87 & 80.87 \\
\hline 21 & 0.000932 & 0.999068 & $96,975.96$ & 90.37 & 53.04 & 74.04 & 71 & 0.043486 & 0.956514 & $63,928.53$ & $2,779.99$ & 10.29 & 81.29 \\
\hline 22 & 0.000973 & 0.999027 & $96,885.60$ & 94.26 & 52.09 & 74.09 & 72 & 0.047834 & 0.952166 & $61,148.54$ & $2,925.00$ & 9.73 & 81.73 \\
\hline 23 & 0.000981 & 0.999019 & $96,791.34$ & 94.91 & 51.14 & 74.14 & 73 & 0.052306 & 0.947694 & $58,223.54$ & $3,045.44$ & 9.20 & 82.20 \\
\hline 24 & 0.000955 & 0.999045 & $96,696.43$ & 92.33 & 50.19 & 74.19 & 74 & 0.056931 & 0.943069 & $55,178.10$ & $3,141.34$ & 8.68 & 82.68 \\
\hline 25 & 0.000919 & 0.999081 & $96,604.09$ & 88.75 & 49.24 & 74.24 & 75 & 0.063283 & 0.936717 & $52,036.76$ & 3,293.05 & 8.17 & 83.17 \\
\hline 26 & 0.000905 & 0.999095 & $96,515.35$ & 87.31 & 48.28 & 74.28 & 76 & 0.071007 & 0.928993 & $48,743.71$ & $3,461.13$ & 7.69 & 83.69 \\
\hline 27 & 0.000906 & 0.999094 & $96,428.03$ & 87.40 & 47.33 & 74.33 & 77 & 0.078297 & 0.921703 & $45,282.58$ & $3,545.49$ & 7.24 & 84.24 \\
\hline 28 & 0.000924 & 0.999076 & $96,340.63$ & 89.00 & 46.37 & 74.37 & 78 & 0.084963 & 0.915037 & $41,737.09$ & $3,546.13$ & 6.81 & 84.81 \\
\hline 29 & 0.000957 & 0.999043 & $96,251.64$ & 92.11 & 45.41 & 74.41 & 79 & 0.090677 & 0.909323 & $38,190.96$ & $3,463.05$ & 6.40 & 85.40 \\
\hline 30 & 0.000973 & 0.999027 & $96,159.52$ & 93.56 & 44.45 & 74.45 & 80 & 0.099237 & 0.900763 & $34,727.91$ & $3,446.30$ & 5.99 & 85.99 \\
\hline 31 & 0.000978 & 0.999022 & $96,065.96$ & 94.00 & 43.50 & 74.50 & 81 & 0.111418 & 0.888582 & $31,281.61$ & $3,485.35$ & 5.59 & 86.59 \\
\hline 32 & 0.001010 & 0.998990 & $95,971.97$ & 96.93 & 42.54 & 74.54 & 82 & 0.123215 & 0.876785 & $27,796.27$ & $3,424.93$ & 5.23 & 87.23 \\
\hline 33 & 0.001068 & 0.998932 & $95,875.03$ & 102.38 & 41.58 & 74.58 & 83 & 0.133970 & 0.866030 & $24,371.34$ & $3,265.03$ & 4.90 & 87.90 \\
\hline 34 & 0.001152 & 0.998848 & $95,772.66$ & 110.32 & 40.62 & 74.62 & 84 & 0.142406 & 0.857594 & $21,106.31$ & $3,005.67$ & 4.57 & 88.57 \\
\hline 35 & 0.001202 & 0.998798 & $95,662.34$ & 115.02 & 39.67 & 74.67 & 85 & 0.153331 & 0.846669 & $18,100.64$ & $2,775.38$ & 4.25 & 89.25 \\
\hline 36 & 0.001236 & 0.998764 & $95,547.31$ & 118.05 & 38.72 & 74.72 & 86 & 0.170400 & 0.829600 & $15,325.25$ & $2,611.42$ & 3.93 & 89.93 \\
\hline 37 & 0.001320 & 0.998680 & $95,429.26$ & 125.95 & 37.77 & 74.77 & 87 & 0.189071 & 0.810929 & $12,713.84$ & $2,403.82$ & 3.64 & 90.64 \\
\hline 38 & 0.001456 & 0.998544 & $95,303.31$ & 138.72 & 36.81 & 74.81 & 88 & 0.208788 & 0.791212 & $10,310.01$ & $2,152.61$ & 3.37 & 91.37 \\
\hline 39 & 0.001643 & 0.998357 & $95,164.59$ & 156.37 & 35.87 & 74.87 & 89 & 0.227740 & 0.772260 & $8,157.41$ & $1,857.76$ & 3.12 & 92.12 \\
\hline 40 & 0.001769 & 0.998231 & $95,008.22$ & 168.05 & 34.93 & 74.93 & 90 & 0.241418 & 0.758582 & $6,299.64$ & $1,520.85$ & 2.90 & 92.90 \\
\hline 41 & 0.001861 & 0.998139 & $94,840.17$ & 176.49 & 33.99 & 74.99 & 91 & 0.253833 & 0.746167 & $4,778.79$ & $1,213.02$ & 2.66 & 93.66 \\
\hline 42 & 0.002048 & 0.997952 & $94,663.67$ & 193.87 & 33.05 & 75.05 & 92 & 0.271552 & 0.728448 & $3,565.78$ & 968.29 & 2.39 & 94.39 \\
\hline 43 & 0.002331 & 0.997669 & $94,469.81$ & 220.18 & 32.12 & 75.12 & 93 & 0.302863 & 0.697137 & $2,597.48$ & 786.68 & 2.10 & 95.10 \\
\hline 44 & 0.002710 & 0.997290 & $94,249.63$ & 255.42 & 31.19 & 75.19 & 94 & 0.368996 & 0.631004 & $1,810.80$ & 668.18 & 1.80 & 95.80 \\
\hline 45 & 0.003020 & 0.996980 & $93,994.21$ & 283.87 & 30.27 & 75.27 & 95 & 0.444244 & 0.555756 & $1,142.62$ & 507.6 & 1.55 & 96.55 \\
\hline 46 & 0.003269 & 0.996731 & $93,710.34$ & 306.36 & 29.36 & 75.36 & 96 & 0.480228 & 0.519772 & 635.02 & 304.95 & 1.40 & 97.40 \\
\hline
\end{tabular}




\begin{tabular}{rrrrrrrrrrrrrr}
\hline $\mathbf{4 7}$ & 0.003630 & 0.996370 & $93,403.98$ & 339.04 & 28.46 & 75.46 & $\mathbf{9 7}$ & 0.501158 & 0.498842 & 330.06 & 165.41 & 1.23 & 98.23 \\
$\mathbf{4 8}$ & 0.004104 & 0.995896 & $93,064.94$ & 381.93 & 27.56 & 75.56 & $\mathbf{9 8}$ & 0.540448 & 0.459552 & 164.65 & 88.98 & 0.96 & 98.96 \\
$\mathbf{4 9}$ & 0.004693 & 0.995307 & $92,683.01$ & 435.00 & 26.67 & 75.67 & $\mathbf{9 9}$ & 1.000000 & 0.000000 & 75.67 & 75.67 & 0.50 & 99.50 \\
\hline
\end{tabular}

$l_{x}$ is the survivorship function, namely the number of persons alive at age $x$. For example, of the original 100,000 females in the hypothetical cohort, $l_{50}=96,639$ (or $96.639 \%$ ) live to age 50 . These values are computed recursively from the $m_{x}$ values using the following formula:

$$
l_{x+1}=l_{x} e^{-m_{x}} \quad(1)
$$

with $l_{0}$, the radix of the table, arbitrarily set to 100,000 . For example

$$
l_{2}=l_{1} e^{-m_{1}}=99,184 e^{-0.000278}=99,156(1)
$$

$\boldsymbol{d}_{x}$ is number of deaths in the interval $(x, x+1)$ for persons alive at age $x$, computed as follows:

$$
d_{x}=l_{x}-l_{x+1}(2)
$$

For example, of the $l_{50}=96,639$ persons alive at age 50

$$
d_{50}=l_{50}-l_{51}=96,639-96,383=256(2)
$$

Thus, according to the formula, 256 persons died prior to age 51. $\boldsymbol{q}_{x}$ is the probability of dying at age $x$, also known as the (age-specific) risk of death. Generally, these are derived using the formula

$q_{x}=1-e^{-m_{x}}=1-p_{x}(3)$

under the assumption that the instantaneous mortality rate, or force of mortality, remains constant throughout the age interval from $x$ to $x+1$, whereas $p_{x}$ is the probability of living at age $x$. By construction, $q_{x}$ is also equal to $d_{x} / l_{x}$. Thus, for example

$q_{50}=\frac{d_{50}}{l_{50}}=\frac{256}{96,639}=0.002650(3)$

$m_{x}$ is the mortality rate at age $x$. Generally, these quantities are estimated from the data and are the sole input to the life table. That is, all other quantities are determined once the $m_{x}$ values are specified. By construction

$$
m_{x}=\frac{d_{x}}{L_{x}}(4)
$$

where the number of deaths at age $x$ is divided by the number of person-years at risk at age $x$. Note that the mortality rate, $m_{x}$, and the probability of death, $q_{x}$, are not identical. For a one-year interval, they will be close in value, but $m_{x}$ will always be larger.

$L_{x}$ is midpoint survivorship, i.e., total number of person-years lived by the cohort from age $x$ to $x+1$ (Princee, 2016). This is the sum of years lived by the $l_{x+1}$ persons who survive the interval, and $d_{x}$ persons who die during the interval. The former contribute exactly 1 year each, while the latter contribute, on average, approximately half a year, so that

$L_{x}=l_{x+1}+0.5 \times d_{x}(5)$

This approximation assumes that deaths occur, on average, half way in the age interval $x$ to $x+1$.

It is also possible to view $L_{x}$ as the average number of persons alive during the interval $x$ to $x+1$ :

$L_{x}=l_{x+1}+0.5 \times d_{x}=l_{x+1}+0.5 \times\left(l_{x}-l_{x+1}\right)=\frac{l_{x}+l_{x+1}}{2}(5)$

$T_{x}$ is total number of person-years lived by the cohort from age $x$ until all members of the cohort have died. This is the sum of numbers in the Lx column from age $x$ to the last row in the table. $\boldsymbol{e}_{x}$ is the (remaining) life expectancy of persons alive at age $x$, computed as follows:

$$
e_{x}=\frac{T_{x}}{l_{x}}(6)
$$

For example, at age 50 , the life expectancy is

$e_{50}=\frac{T_{50}}{l_{50}}=\frac{2,873,915}{96,639}=29.74(6)$

Finally, $e_{x}$ at $x$ is average life expectancy for persons at age $x$ :

$e_{x}$ at $x=e_{x}+x(7)$

For example, at age 50 , the life expectancy is

$e_{50}$ at $50=e_{50}+50=29.74+50=79.74(7)$

\subsection{Minimum Amount of One-Time Endowment Needed to Cover Lifetime Expenses}

The minimum amount of the one-time endowment needed to cover lifetime expenses is assumed to be equal to the actuarial present value of monthly payments determined by the Ministry of Family and Social Policies. In this calculation, we prefer to use real rather than nominal values in order to avoid estimation of annual inflation figures. This necessitates the use of the real rate of 
return in the computation of discount factors. Accordingly, we have also implicitly assumed that the costs of taking care of elderly and, hence, the monthly payments increase at the rate of inflation. We compute the actuarial present value of $€ 1$ spent each year as long as the elderly person lives. This actuarial present value corresponds to the minimum multiple needed to be used in the computation of one-time endowment. For instance, if the actuarial present value turns out to be 10, then the nursing home needs to demand a one-time endowment that is equivalent to an upfront payment for at least 10 years.

Under these assumptions, the actuarial present value of $€ 1$ spent each year as long as the elderly lives is given by (MIT, 2005):

Actuarial Present Value of $€ 1=\sum_{k=1}^{\omega-x}{ }_{k} L_{x} v^{(k-0.5)}(8)$

where

$\omega=$ highest age in the mortality table; ${ }^{1}$

$x=$ current age of the elderly;

${ }_{k} \boldsymbol{L}_{x}=$ probability of spending $€ 1$ for an elderly who is at the age of $x$ in the $k$ th year after her/his acceptance to the nursing home;

$v=$ discount factor;

${ }_{k} L_{x}$ is given by

$$
{ }_{k} L_{x}=\frac{L_{x+k-1}}{l_{x}}
$$

i.e., the average number of persons alive during the interval $x+k-1$ to $x+k$ divided by the number of persons alive at age $x$.

It is required by law to be over 60 years old to apply to live in a nursing home. For this reason, our results are tabulated for ages 60 and above. As an example, for ages 60 and 70, we show the average number of persons alive during the interval $x+k-1$ to $x+k$, the number of persons alive at age $x$, the probability of spending $€ 1$ for an elderly who is at the age of $x$ in the $k$ th year after her/his acceptance to the nursing home, the discount factor for each year $k$, and the calculated actuarial present value of $€ 1$ spent each year as long as the elderly lives in Table 3 and Table 4. 
Table 3. Actuarial Present Value Calculation for Age 60

\begin{tabular}{|c|c|c|c|c|c|c|c|c|c|}
\hline \multirow{2}{*}{$\mathbf{k}$} & \multicolumn{2}{|c|}{$\mathbf{L}_{60+k-1}$} & \multicolumn{2}{|c|}{$\mathbf{I}_{60}$} & \multicolumn{2}{|c|}{${ }_{k} \mathbf{L}_{60}$} & \multirow{2}{*}{$\mathbf{v}^{k}$} & \multicolumn{2}{|c|}{ Actuarial Present Value } \\
\hline & Female & Male & Female & Male & Female & Male & & Female & Male \\
\hline $\mathbf{1}$ & 92,220 & 83,901 & 92,556 & 84,526 & 0.9964 & 0.9926 & 0.9901 & 16.56 & 14.36 \\
\hline 2 & 91,530 & 82,609 & 92,556 & 84,526 & 0.9889 & 0.9773 & 0.9707 & & \\
\hline 3 & 90,783 & 81,220 & 92,556 & 84,526 & 0.9808 & 0.9609 & 0.9517 & & \\
\hline 4 & 89,942 & 79,714 & 92,556 & 84,526 & 0.9718 & 0.9431 & 0.9330 & & \\
\hline 5 & 88,970 & 78,070 & 92,556 & 84,526 & 0.9613 & 0.9236 & 0.9147 & & \\
\hline 6 & 87,863 & 76,282 & 92,556 & 84,526 & 0.9493 & 0.9025 & 0.8968 & & \\
\hline 7 & 86,644 & 74,361 & 92,556 & 84,526 & 0.9361 & 0.8797 & 0.8792 & & \\
\hline 8 & 85,307 & 72,307 & 92,556 & 84,526 & 0.9217 & 0.8554 & 0.8620 & & \\
\hline 9 & 83,815 & 70,109 & 92,556 & 84,526 & 0.9056 & 0.8294 & 0.8451 & & \\
\hline 10 & 82,129 & 67,756 & 92,556 & 84,526 & 0.8873 & 0.8016 & 0.8285 & & \\
\hline 11 & 80,239 & 65,234 & 92,556 & 84,526 & 0.8669 & 0.7718 & 0.8123 & & \\
\hline 12 & 78,167 & 62,539 & 92,556 & 84,526 & 0.8445 & 0.7399 & 0.7963 & & \\
\hline 13 & 75,912 & 59,686 & 92,556 & 84,526 & 0.8202 & 0.7061 & 0.7807 & & \\
\hline 14 & 73,448 & 56,701 & 92,556 & 84,526 & 0.7936 & 0.6708 & 0.7654 & & \\
\hline 15 & 70,751 & 53,607 & 92,556 & 84,526 & 0.7644 & 0.6342 & 0.7504 & & \\
\hline 16 & 67,784 & 50,390 & 92,556 & 84,526 & 0.7324 & 0.5962 & 0.7357 & & \\
\hline 17 & 64,529 & 47,013 & 92,556 & 84,526 & 0.6972 & 0.5562 & 0.7213 & & \\
\hline 18 & 61,033 & 43,510 & 92,556 & 84,526 & 0.6594 & 0.5148 & 0.7071 & & \\
\hline 19 & 57,368 & 39,964 & 92,556 & 84,526 & 0.6198 & 0.4728 & 0.6933 & & \\
\hline 20 & 53,609 & 36,459 & 92,556 & 84,526 & 0.5792 & 0.4313 & 0.6797 & & \\
\hline 21 & 49,751 & 33,005 & 92,556 & 84,526 & 0.5375 & 0.3905 & 0.6663 & & \\
\hline 22 & 45,730 & 29,539 & 92,556 & 84,526 & 0.4941 & 0.3495 & 0.6533 & & \\
\hline 23 & 41,582 & 26,084 & 92,556 & 84,526 & 0.4493 & 0.3086 & 0.6405 & & \\
\hline 24 & 37,428 & 22,739 & 92,556 & 84,526 & 0.4044 & 0.2690 & 0.6279 & & \\
\hline 25 & 33,390 & 19,603 & 92,556 & 84,526 & 0.3608 & 0.2319 & 0.6156 & & \\
\hline 26 & 29,488 & 16,713 & 92,556 & 84,526 & 0.3186 & 0.1977 & 0.6035 & & \\
\hline 27 & 25,639 & 14,020 & 92,556 & 84,526 & 0.2770 & 0.1659 & 0.5917 & & \\
\hline 28 & 21,865 & 11,512 & 92,556 & 84,526 & 0.2362 & 0.1362 & 0.5801 & & \\
\hline 29 & 18,292 & 9,234 & 92,556 & 84,526 & 0.1976 & 0.1092 & 0.5687 & & \\
\hline 30 & 15,044 & 7,229 & 92,556 & 84,526 & 0.1625 & 0.0855 & 0.5576 & & \\
\hline 31 & 12,190 & 5,539 & 92,556 & 84,526 & 0.1317 & 0.0655 & 0.5466 & & \\
\hline 32 & 9,693 & 4,172 & 92,556 & 84,526 & 0.1047 & 0.0494 & 0.5359 & & \\
\hline 33 & 7,503 & 3,082 & 92,556 & 84,526 & 0.0811 & 0.0365 & 0.5254 & & \\
\hline 34 & 5,606 & 2,204 & 92,556 & 84,526 & 0.0606 & 0.0261 & 0.5151 & & \\
\hline 35 & 3,988 & 1,477 & 92,556 & 84,526 & 0.0431 & 0.0175 & 0.5050 & & \\
\hline 36 & 2,646 & 889 & 92,556 & 84,526 & 0.0286 & 0.0105 & 0.4951 & & \\
\hline 37 & 1,589 & 483 & 92,556 & 84,526 & 0.0172 & 0.0057 & 0.4854 & & \\
\hline 38 & 816 & 247 & 92,556 & 84,526 & 0.0088 & 0.0029 & 0.4759 & & \\
\hline 39 & 312 & 120 & 92,556 & 84,526 & 0.0034 & 0.0014 & 0.4665 & & \\
\hline 40 & 63 & 38 & 92,556 & 84,526 & 0.0007 & 0.0004 & 0.4574 & & \\
\hline
\end{tabular}


Table 4. Actuarial Present Value Calculation for Age 70

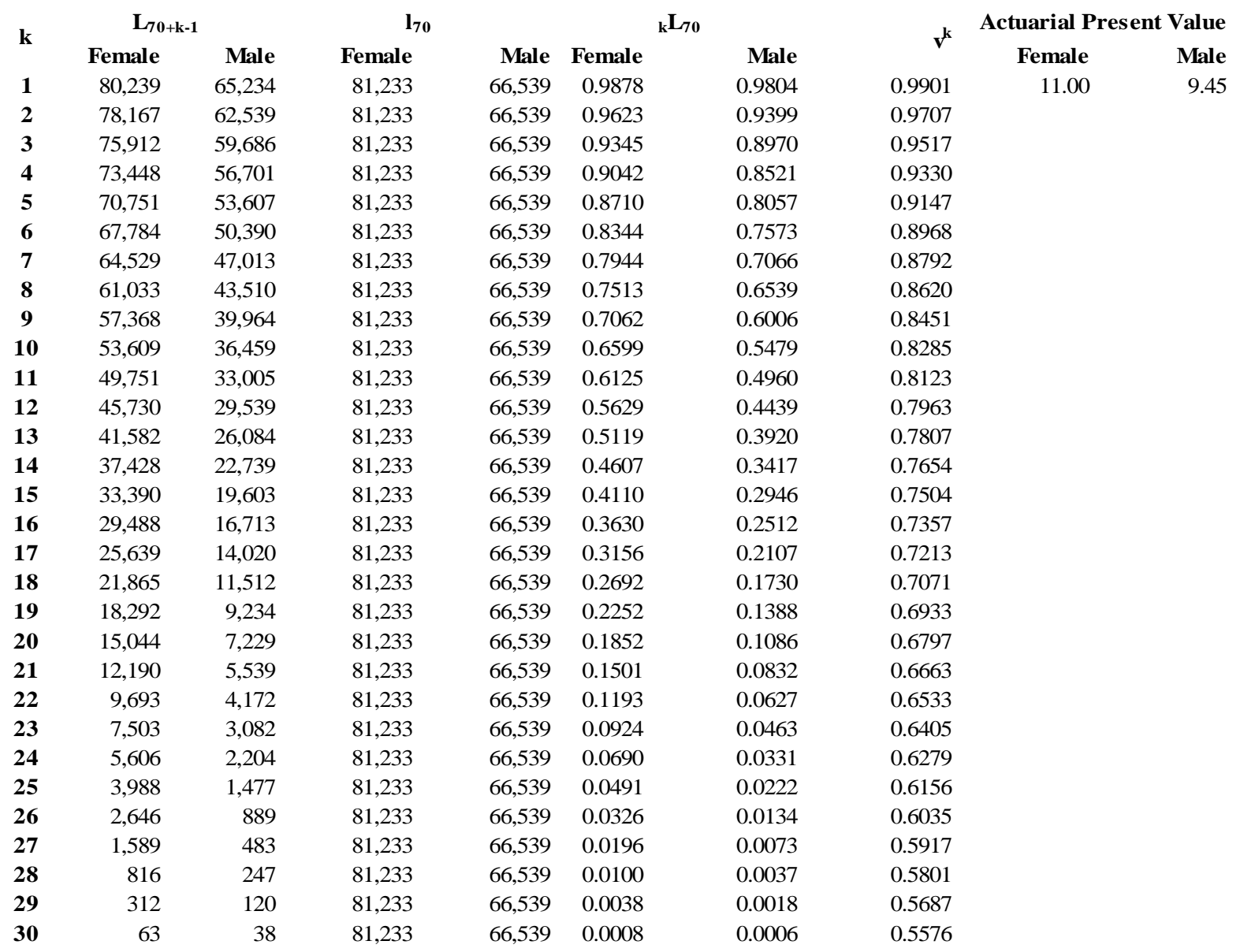

These calculations are performed for all female and male elderly persons above the age of 60 . The results are shown in Table 5.

Table 5. Actuarial Present Value

$\begin{array}{crrrrr}\text { Age } & \text { Female } & \text { Male } & \text { Age } & \text { Female } & \text { Male } \\ \mathbf{6 0} & 16.56 & 14.36 & \mathbf{8 0} & 6.36 & 5.49 \\ \mathbf{6 1} & 16.00 & 13.84 & \mathbf{8 1} & 5.97 & 5.15 \\ \mathbf{6 2} & 15.43 & 13.33 & \mathbf{8 2} & 5.61 & 4.84 \\ \mathbf{6 3} & 14.86 & 12.83 & \mathbf{8 3} & 5.26 & 4.55 \\ \mathbf{6 4} & 14.30 & 12.32 & \mathbf{8 4} & 4.93 & 4.27 \\ \mathbf{6 5} & 13.74 & 11.83 & \mathbf{8 5} & 4.58 & 3.99 \\ \mathbf{6 6} & 13.19 & 11.34 & \mathbf{8 6} & 4.25 & 3.70 \\ \mathbf{6 7} & 12.63 & 10.85 & \mathbf{8 7} & 3.95 & 3.44 \\ \mathbf{6 8} & 12.08 & 10.38 & \mathbf{8 8} & 3.67 & 3.19 \\ \mathbf{6 9} & 11.53 & 9.91 & \mathbf{8 9} & 3.40 & 2.97 \\ \mathbf{7 0} & 11.00 & 9.45 & \mathbf{9 0} & 3.13 & 2.77 \\ \mathbf{7 1} & 10.48 & 9.00 & \mathbf{9 1} & 2.84 & 2.55 \\ \mathbf{7 2} & 9.97 & 8.56 & \mathbf{9 2} & 2.54 & 2.31 \\ \mathbf{7 3} & 9.46 & 8.14 & \mathbf{9 3} & 2.24 & 2.03 \\ \mathbf{7 4} & 8.97 & 7.72 & \mathbf{9 4} & 1.93 & 1.74 \\ \mathbf{7 5} & 8.49 & 7.31 & \mathbf{9 5} & 1.63 & 1.51 \\ \mathbf{7 6} & 8.03 & 6.91 & \mathbf{9 6} & 1.33 & 1.37 \\ \mathbf{7 7} & 7.60 & 6.54 & \mathbf{9 7} & 1.03 & 1.20 \\ \mathbf{7 8} & 7.18 & 6.19 & \mathbf{9 8} & 0.74 & 0.95 \\ \mathbf{7 9} & 6.77 & 5.84 & \mathbf{9 9} & 0.50 & 0.50\end{array}$


Our first and most important observation is that age has the highest impact on the minimum amount of the initial one-time endowment required to cover lifetime expenses. At age 60, this corresponds to an upfront payment for 16.56 and 14.36 years for female and male elderly persons, respectively. Similarly, these required payments correspond to 11.00 and 9.45 years for age 70, respectively, for female and male elderly persons. These findings also demonstrate that gender has a considerable impact on the required minimum initial endowment.

For example, nursing homes operated by the Turkish Red Crescent required a monthly payment of $€ 1,750$ for a single room in 2017. Using this amount as a basis for monthly costs, the minimum amount of initial one-time endowment should be at least $€ 347,714$ and $€ 301,467$ for female and male elderly persons who are at the age of 60 . For age 70 , these figures correspond to $€ 231,077$ and $£ 198,346$. However, these nursing homes demand a flat rate of $£ 210,000$ without consideration of the age or gender of the elderly resident. Hence, a customary endowment at the time of acceptance that covers 10 years of expenses is insufficient for female elderly below the age of 71 and male elderly below the age of 68 .

\section{Discussion}

Two different payment methods are available for elderly people admitted to a nursing home in Turkey. Regardless of method, the payment amount will be of interest to all stakeholders, such as the elderly and their relatives, nursing institutions, governments, etc. Currently, individual-specific payment amounts are not usually computed. This is understandable to some extent for social and moral reasons. Nevertheless, it is highly probable that innovations in payment methods for nursing homes will occur in the near future given the aging of the population in many countries along with increasing expectations for service differentiation and growing financial pressures on individuals, nursing institutions, and publicly financed social security systems.

Applying a flat rate of initial endowment regardless of age and gender is inequitable yet potentially threatening to the solvency of nursing institutions. As demonstrated, the life expectancy for a 75 -year-old man is 8.17 years, which is less than the half of the life expectancy for a 60 -year old man, which is 17.62 years. For this reason, taking a flat rate from elderly persons above a certain age is unfair. For social reasons, it may be desirable to provide a transfer of resources from the young to elderly and/or from the healthy to the sick. However, failure to take factors such as age and gender into account in pricing may seriously jeopardize the fairness and sustainability of elderly services. For this reason, it is crucial to use more equitable and fair payment models for nursing homes.

Other factors that affect the care costs of the elderly have also been examined in the literature. Welch et al. examined the impact of Alzheimer's disease on the cost and length of stay in nursing homes. The median length of stay for Alzheimer's patients was 2.75 years, over 10 times the national median length of stay for all diagnoses, and nursing home charges were estimated to be between $\$ 35,000$ and $\$ 52,000$ per patient (Welch et all, 1992). Other studies focused on the impact of institutional characteristics on costs and pricing rather than the individual characteristics of the elderly. For instance, in studies conducted by Birnbaum et al. and McKay, the effects of chain ownership on nursing home costs have been examined. Chain ownership resulted in lower costs due to economies of scale (McKay, 1991). Hazra, Rudisill and Gulliford have investigated the determinants of health care costs in the senior elderly and found that annual health care costs increased from 80 years ( $£ 2972$ in men, $£ 2603$ in women) to 97 (men; $£ 4721$ ) or 98 years (women; $£ 3963$ ), before declining. Costs were significantly elevated in the last year of life but this effect declined with age, from $£ 10,027$ in younger octogenarians to $£ 7021$ in centenarians. This decline was steeper in participants with comorbidities or impairments; $£ 14,500$ for $80-84$-year-olds and $£ 6752$ for centenarians with 7+ impairment (Hazra, Rudisill and Gulliford, 2018).

Kemper and Murtaugh found that the probability of nursing home use increases sharply with age at death: $17 \%$ for age 65 to $74,36 \%$ for age 75 to 84 , and $60 \%$ for age 85 to 94 . According to this study, it is projected that more women than men will enter nursing homes (52\% vs. $33 \%$ ), and among them, more women than men will have total lifetime nursing home use of 5 years or more (25\% vs. 13\%) (Kemper and Murtaugh, 1991). These results are similar to ours. Kemper and Murtaugh also 
examined the impact of ethnic differences on the cost and length of staying in nursing homes and found significant differences between persons of black and white ethnic backgrounds.

Some studies examined the effect of disability status on mortality in addition to the effects of age and gender. For example, in the simulation study conducted by Rasoanaivo, disabled persons were assumed to have a mortality rate three times higher than that of the whole population (Rasoanaivo, 2001). In a study conducted by Lew and Garfınkel, cigarette use and obesity were found to be significant factors affecting mortality (Lew and Garfınkel, 1987). In an other study Furlan and Fehlings have examined the impact of age on mortality, impairment, and disability among adults with acute traumatic spinal cord injury and found that mortality rates among older people ( $\geq 65$ years) were significantly greater than those of younger individuals at 6 weeks, at 6 months, and at 1 year following spinal cord injury. Among survivors, age was not significantly correlated with motor recovery or change in pain scores in the acute and chronic stages after spinal cord injury based on regression analyses adjusted for major confounders (Furlan and Fehlings, 2009).

It should be noted that some macroeconomic assumptions such as the real rate of return may also have a significant impact on the results of this study. Considering the low-interest-rate environment existing after the global crisis of 2008 , a $2 \%$ real rate of return may be too optimistic. A lower real rate of return will automatically translate into a required higher initial one-time endowment.

\section{Conclusion and Evaluation}

Some reasons may make it difficult to enforce age and, particularly, gender differences in pricing. However, it is imperative for nursing institutions to take these factors into account even if a flat rate is charged. In a hypothetical setting, an institution where the occupants are mostly relatively younger women should charge a much higher flat rate compared to another institution where the occupants are relatively older men. The administration of such a pricing policy is critical for the financial sustainability of nursing institutions.

Hence, the results of this study have two potential uses. First, it may be possible to use individual-specific pricing in a social setting where use of factors such as age and gender is acceptable. Second, even if individual-specific pricing is not possible for social and/or legal reasons, nursing institutions can utilize similar models for financial planning purposes to ensure long-term sustainability. The model can be extended to account for additional resources such as extra physical space and additional employees for varying demographic compositions of nursing home inhabitants.

For future studies, we intend to explore the impact of other individual-specific factors such as smoking and drinking habits and/or obesity if sufficient amounts of reliable data can be obtained. We also plan to elaborate on the impact of macroeconomic factors such as the real rate of return.

\section{References}

Ardahan, M. (2010). Old age and nursing home. Family and Society, 5(20), 25-32

Çadır, M. (2017). The transformation of social services for the elderly in England: an overview of the nursing home sector. Journal of Social Policy Studies, 17(39), 9-32.

Furlan, J. C., \& Fehlings, M. G. (2009). The impact of age on mortality, impairment, and disability among adults with acute traumatic spinal cord injury. Journal of neurotrauma, 26(10), 17071717. https://doi.org/10.1089/neu.2009.0888

Glass, A.P. (1991). Nursing home quality: a framework for analysis. Journal of Applied Gerontology, 10(1),5-18.

Gokbunar, A.R., Ugur, A., \& Duramaz, S. (2016). The importance of public policies of the decreasing in health spending for elderly population. The International Journal of Economic and Social Research, 12(1),109-122. 
Hazra, N.C., Rudisill, C. \& Gulliford, M.C. (2018). Determinants of health care costs in the senior elderly: age, comorbidity, impairment, or proximity to death?. Eur J Health Econ 19, 831-842 https://doi.org/10.1007/s10198-017-0926-2

Hacettepe University Actuarial Sciences Department Documents (2010), Turkey Mortality Project: Turkey Life Tables. http://www.aktuerya.hacettepe.edu.tr/TurkiyeHayatTablolari.php 10.01.2018.

Kemper, P., \& Murtaugh, C.M. (1991). Lifetime use of nursing home care. J of Medicine. 324, 595-600. Lau, D., Kasper, J., Potter, D., Lyles, A., \& Bennett, R. (2005). Hospitalization and death associated with potentially inappropriate medication prescriptions among elderly nursing home residents. Arch Internal Medicine, 165, 68-75.

Lew, E., \& Garfinkel, L. (1987). Differences in mortality and longevity by sex, smoking habits and health status. Transactions of Society of Actuaries, 39, 107-30

McKay, N.L. (1991). The effect of chain ownership on nursing home costs. Health Services Research, 26(1), 109-24.

MIT Department of Biology (2005), Life Tables. http:/ / studylib.net/doc/13541403/life-tables 27. 01. 2018

Official Newspaper of Turkey (2001), Nursing Home And Nursing Home Regulation on The Care and Rehabilitation Centers for the Elderly. General Directorate of Legislation Development and Publication.http://www.mevzuat.gov.tr/Metin.Aspx?MevzuatKod=7.5.4414\&MevzuatIliski=0 \&sourceXmlSearch 20.01. 2018

Princee, F. P. G. (2016): Exploring studbooks for wildlife management and conservation. Springer International Publishing

Rasoanaivo, G. R. (2001): Stochastic modeling of long term care insurance. University of Connecticut Press

Strauss, D., \& Shavelle, R. (2018). The life table, life expectancy. http://www.lifeexpectancy.org/lifetable.shtml (27. 01. 2018)

Wagner, C., Wal, G., Groenewegen, P.P., \& Bakker, D.H. (2001). The efectiveness of quality systems in nursing homes: a review. Quality in Health Care, 10,211-217.

Welch, H.G., Walsh, J.S., \& Larson, E.B. (1992). The Cost of institutional care in alzheimer's disease: nursing home and hospital use in a prospective cohort. J of the American Geriatrics Society, 40(3), 221-24.

World Health Organization (2016), Life expectancy and Healthy Life Expecancy Data by WHO Region. Global Health Observatory Data Repository. WHO http://apps.who.int/gho/data/view.main.SDG2016LEXREGv?lang=en 22. 01. 2018

\footnotetext{
Appendix A - R Script

setwd("C:/Users/USER/Downloads")

\# read data from csv files which include mortality tables for female and male elderly

femaledata=read.csv(file="kadinmortalite0.txt",header=TRUE,sep=";")

maledata $=$ read.csv(file="erkekmortalite0.txt",header=TRUE, sep $=" ; ")$

\# assumption for real rate of return

rror $=.02$

\# for each age $x$, calculate

\# Lx+k-1(average number of persons alive during the interval $\mathrm{x}+\mathrm{k}-1$ to $\mathrm{x}+\mathrm{k}$ ),

\# kLx (probability of spending $€ 1$ for an elderly who is at the age of $x$ in the k-th year

\# after her/his acceptance to the nursing home)

\# vk (discount factor)

\# APV (actuarial present value)

$\operatorname{Lxk} 1=\operatorname{array}(\operatorname{dim}=\mathrm{c}(2,99,40))$

$\mathrm{kLx}=\operatorname{array}(\operatorname{dim}=\mathrm{c}(2,99,40))$
} 


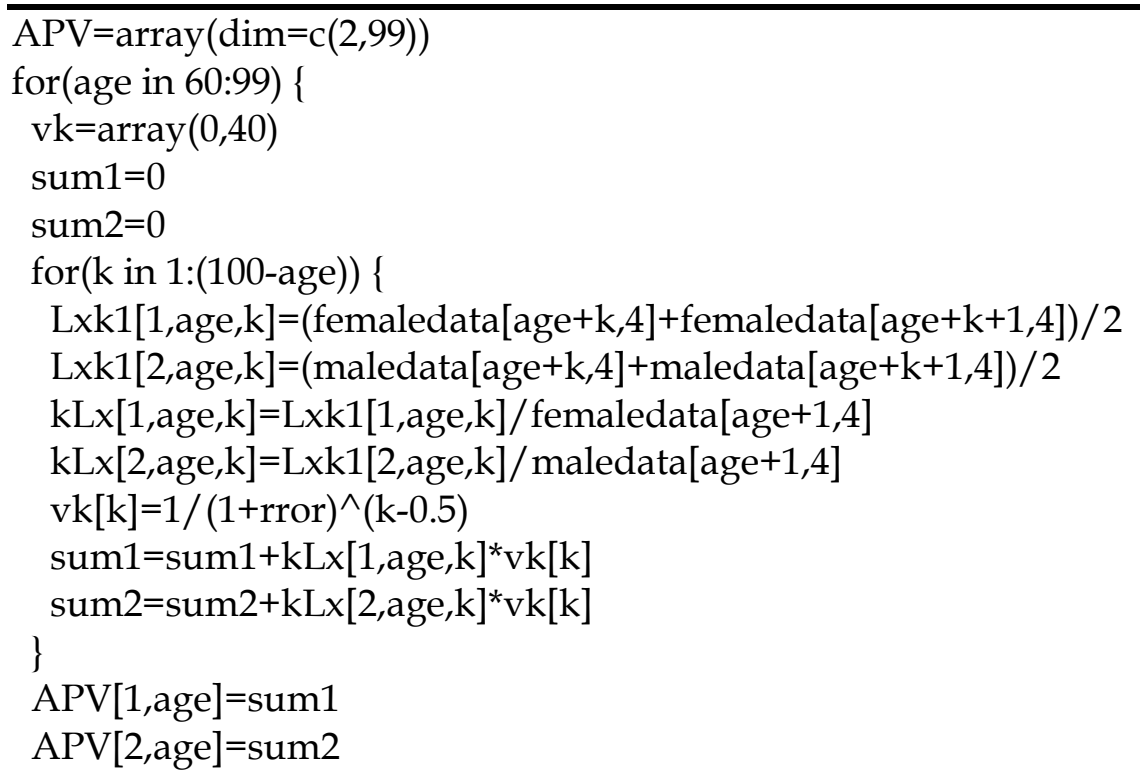

